\title{
Radiation dose reduction in CT-guided sacroiliac joint injections to levels of pulsed fluoroscopy: a comparative study with technical considerations
}

This article was published in the following Dove Press journal:

Journal of Pain Research

10 August 2012

Number of times this article has been viewed

\author{
Juraj Artner \\ Balkan Cakir \\ Heiko Reichel \\ Friederike Lattig \\ Department of Orthopaedic Surgery, \\ University of Ulm, RKU, Germany
}

Background: The sacroiliac (SI) joint is frequently the primary source of low back pain. Over the past decades, a number of different SI injection techniques have been used in its diagnosis and therapy. Despite the concerns regarding exposure to radiation, image-guided injection techniques are the preferred method to achieve safe and precise intra-articular needle placement. The following study presents a comparison of radiation doses, calculated for fluoroscopy and CT-guided SI joint injections in standard and low-dose protocol and presents the technical possibility of CT-guidance with maximum radiation dose reduction to levels of fluoroscopicguidance for a precise intra-articular injection technique.

Objective: To evaluate the possibility of dose reduction in CT-guided sacroiliac joint injections to pulsed-fluoroscopy-guidance levels and to compare the doses of pulsed-fluoroscopy-, CT-guidance, and low-dose CT-guidance for intra-articular SI joint injections.

Study design: Comparative study with technical considerations.

Methods: A total of 30 CT-guided intra-articular SI joint injections were performed in January 2012 in a developed low-dose mode and the radiation doses were calculated. They were compared to 30 pulsed-fluoroscopy-guided SI joint injections, which were performed in the month before, and to five injections, performed in standard CT-guided biopsy mode for spinal interventions. The statistical significance was calculated with the SPSS software using the Mann-Whitney $U$-Test. Technical details and anatomical considerations were provided.

Results: A significant dose reduction of average $94.01 \%$ was achieved using the low-dose protocol for CT-guided SI joint injections. The radiation dose could be approximated to pulsedfluoroscopy-guidance levels.

Conclusion: Radiation dose of CT-guided SI joint injections can be decreased to levels of pulsed fluoroscopy with a precise intra-articular needle placement using the low-dose protocol. The technique is simple to perform, fast, and reproducible.

Keywords: sacroiliac joint pain, computed tomography, guided injections, low-dose protocol, sacroiliac joint injection, low back pain, radiation dose

\section{Introduction}

The sacroiliac (SI) joint has been implicated as the primary source of pain in $10 \%-26.6 \%$ of patients with chronic low back pain. ${ }^{1,2}$ Whereas historical and physical examination findings have been previously advocated as useful tools in identifying patients with SI joint pain, more recent studies have demonstrated that they have limited diagnostic value. ${ }^{3,4}$ Blind, fluoroscopy-, and CT-guided SI joint injection techniques are well described in literature for the diagnosis and treatment of pain originating from SI joints. ${ }^{5-7}$ The purpose of image-guidance in spinal injections is to achieve
Correspondence: Juraj Artner Department of Orthopaedic Surgery, University of Ulm, RKU, Oberer Eselsberg 45, 8908I Ulm, Germany Tel +49 73I I775III

Email j.artner@gmail.com 
safe and precise needle placement and accurate delivery of the injected solution, but the intra-articular needle placement can become problematic even in fluoroscopic-guidance due to the complex orientation and asymmetric anatomy of the SI joint.

Computed tomographic (CT) guidance for injections into the SI joint is the preferred method for some authors for precise intra-articular needle placement because of the complex configuration of the joint. ${ }^{6}$ Long-lasting effects of intra-articular corticoids and TNF-alpha-inhibitors have been described for CT-guided SI injections in patients with sacroiliitis. ${ }^{89}$ Despite the precise intra-articular injection of medications in CT-guidance, radiation exposure remains a serious concern. The aim of the following study was to determine the possibility of rapid radiation dose reduction in intra-articular CT-guided SI joint injections to levels of fluoroscopy without a loss of accuracy in needle placement.

\section{Materials and methods}

A total of 30 CT-guided intra-articular sacroiliac joint injections were performed in January 2012 using a new technique in low-dose mode; the radiation doses were calculated. Inclusion criteria were: inpatient adults, suffering from low-back pain with irradiation to the buttocks, and positive SI joint provocation testing. Patients were excluded if they did not sign the informed consent, had an absolute indication for surgery, were non-adults, or were suffering from coagulopathy, gravidity, or infection. The results were compared to 30 pulsed-fluoroscopy-guided SI joint injections, which were performed during the month prior, and to five injections, performed in standard CT-guided biopsy mode for spinal interventions in the past year.

CT-guided injections were performed by one experienced interventionalist, using the SOMATOM Emotion CT-scanner (syngoCT 2009E, 16-slice solution; Siemens Medical Solutions AG, Erlangen, Germany). The standard intervention mode, used in past SI joint injections consisted of topogram acquisition (120 mA, $130 \mathrm{kV})$, spiral scan (80 mA, $130 \mathrm{kV})$, and biopsy mode $(50 \mathrm{~mA}, 130 \mathrm{kV})$. The low-dose protocol consisted of topographic marking of the SI joint height based on anatomic landmarks (see anatomical and technical considerations) and the biopsy mode with reduced energy and tube current $(50 \mathrm{~mA}, 80 \mathrm{kV})$. Fluoroscopy-guided injections were performed using the AXIOM Luminos dRF (Siemens Medical Solutions AG) in pulsed fluoroscopy mode $(\mathrm{f} /$ second $=7.5)$ for spinal interventions $(60 \mathrm{~mA}, 75-80 \mathrm{kV})$.

Statistical analyses were performed using SPSS statistical software (v 17.0; IBM, Armonk, NY). Descriptive and graphic analyses were provided for the radiation doses $\left(\mathrm{mGycm}^{2}\right)$ for all groups (CT-guided conventional intervention mode, CT-guided low dose mode, and pulsed-fluoroscopy-guided SI joint injections). The radiation dose calculations of the two groups (pulsed-fluoroscopy vs low-dose CT) were compared with the Mann-Whitney $U$-test $\left(\alpha\right.$ level $=0.05 ; H_{0}: \mathrm{n} 1=\mathrm{n} 2$; $\left.\mathrm{H}_{1}: \mathrm{n} 1 \neq \mathrm{n} 2\right)$.

\section{Anatomical and technical considerations}

The sacroiliac joint is a complex diarthrodial joint, consisting of bilateral asymmetric and incongruent corresponding joint surfaces of the ilium and the sacrum. Its multiplanar orientation, irregular joint gap, facultative accessory auricular surfaces or partial ankylosis make blind and fluoroscopyguided intra-articular injections difficult. ${ }^{10,11}$ The joint is partly synovial and partly a syndesmosis, surrounded by thick dorsal and interosseous ligaments. ${ }^{12}$ Together with the variability in surface orientation, they contribute to joint stability. Because the upper two-thirds of the SI joint become fibrotic during adulthood, most interventionalists prefer the caudal portions of the joint for intra-articular injections. . $^{5,10,13}$

Standard protocols for CT-guided spinal injections usually start with a topogram acquisition. The purpose of topogram acquisition is to provide a gross anatomic orientation and selection of the area of interest for further scanning (spiral scan), reconstruction, and intervention. We modified all of these steps in the following manner:

1. Because the gross anatomic SI joint localization can be estimated by palpable anatomic landmarks, the topogram acquisition as used in standard CT-guided interventions was abandoned. The topogram would result in a high level of radiation without significant gain in information. The SI joint location can be approximated by palpation of the following landmarks: sacrum, sacral cornua, and the posterior superior iliac spine. Based on the palpation of these landmarks, the length of the joint can be estimated. To be more precise, we developed a simple technique: to achieve an injection into the caudal portions of the joint, the patient is placed in prone position and the iliac crests and major trochanters of the femur are palpated bilaterally. A virtual horizontal midline is drawn between these structures. This line marks the height of the lower portions of the SI joints. The correct position of the CT-scanner is achieved when the scanner target-laser overlaps the drawn midline where the joint is expected and the topography is controlled by palpation of the sacral cornua located about 4-5 cm caudal to this line (see Figure $1 \mathrm{~A}-\mathrm{C}$ ). 

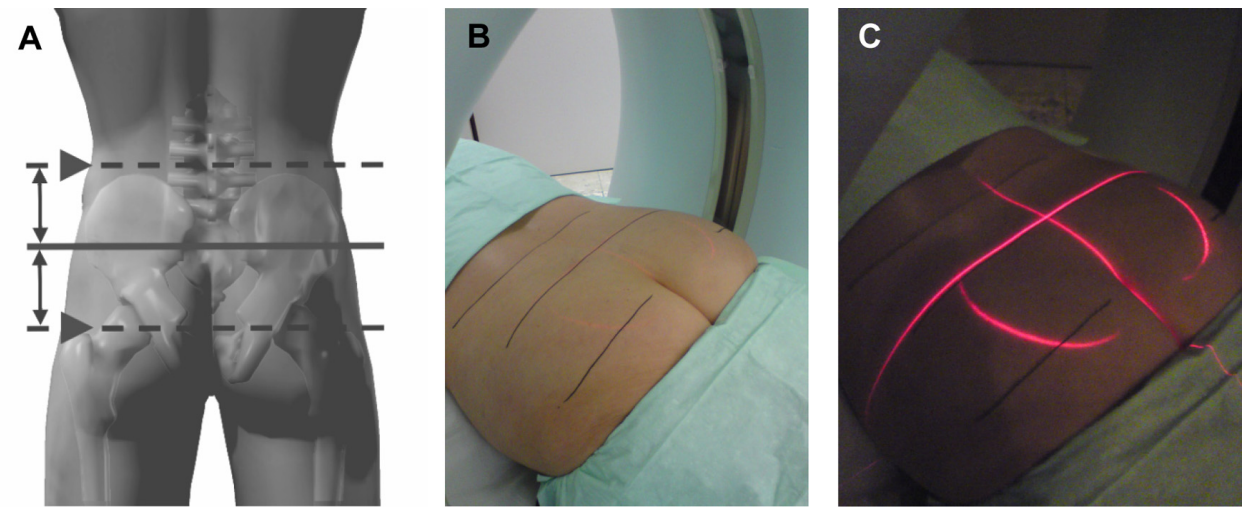

Figure I Preparation for SI joint injection. Based on the anatomic landmarks, the midline between the major trochanter and iliac crest is identified (A) and marked on the skin (B). The target laser of the scanner is brought into an overlapping position with the marked midline (C).

2. No further scanning or reconstructions are needed The biopsy mode is used with reduced energy and tube current to $50 \mathrm{~mA}$ and $80 \mathrm{kV}$ for single shot scans. It is helpful to place both introducer needles (if performing bilateral SI joint injection) so that they cross the marked line (or the overlapping laser line) where the SI joints are expected before the first scan. This step provides a control of the right location (height) and simultaneously allows targeting along the marked line.

3. An average of 3-4 scans is needed for an injection into both SI joints. Despite the reduced image contrast, the bony structures and the needle are still visible even when using the thinnest needle caliber of 29 gauge (see Figures 2 and 3).

\section{Results}

A total of 30 patients ( 22 female and 8 male, age $36-78$ years, mean BMI 28.10, 95\% confidence interval [CI]: 21.75-34.45) underwent bilateral CT-guided SI joint injections in the

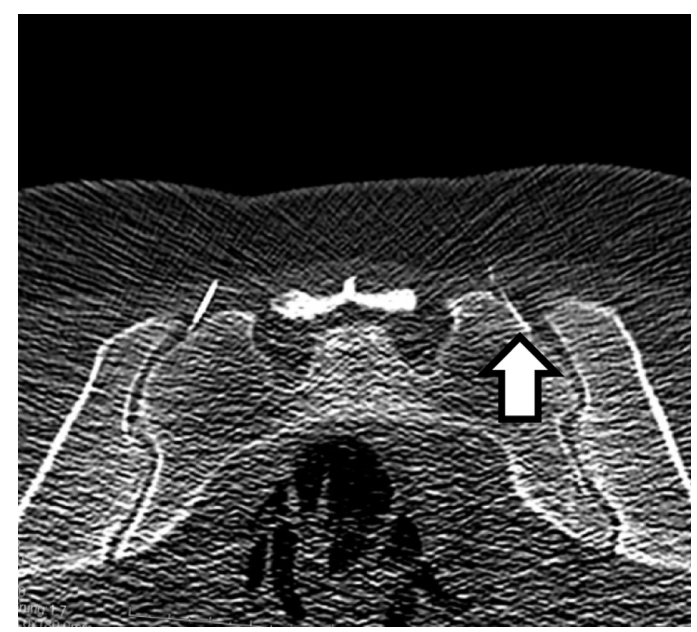

Figure 2 Bilateral CT-guided SI joint injection in low-dose protocol. low-dose protocol in January 2012. The calculated mean radiation dose for these interventions was $4.57 \mathrm{mGycm}^{2}$ (median $=3.890,95 \%$ CI: 4.13-8.24, SD $=1.67)$, using an average of 3-4 scans per procedure. Retrospective data analyses of 30 bilateral pulsed-fluoroscopy-guided SI joint injections, performed in December 2011 presented a mean radiation dose of $3.73 \mathrm{mGycm}^{2}$ (median $=3.895,95 \% \mathrm{CI}=0-6.34$, $\mathrm{SD}=2.58$ ). The five bilateral SI joint injections in conventional CT-intervention mode presented a mean radiation dose of $76.27 \mathrm{mGycm}^{2}$ (95\% CI: 49.31-103.24, SD = 21.72).

Using the established low-dose protocol for CT-guided intra-articular SI joint injections, the mean radiation dose could be reduced to $5.99 \%$ of the level used in conventional CT-guided biopsy mode for spinal interventions, achieving an average dose reduction of $94.01 \%$. The calculated levels of the radiation doses could be approximated to the levels of pulsed fluoroscopy-guidance (see Figures 4A and B). When comparing the calculated radiation doses between the

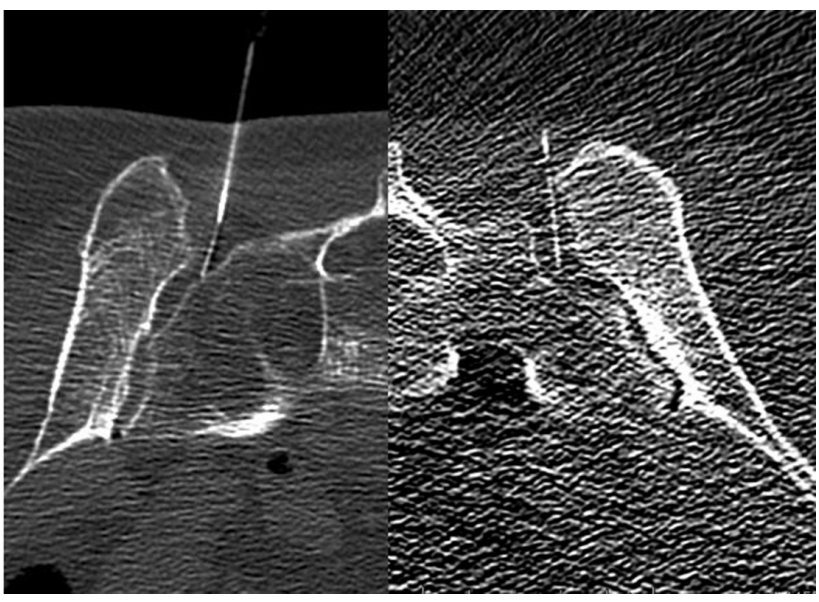

Figure 3 Demonstrations of image quality difference in conventional CT-guidance for spinal interventions (left) and low-dose CT (right). 


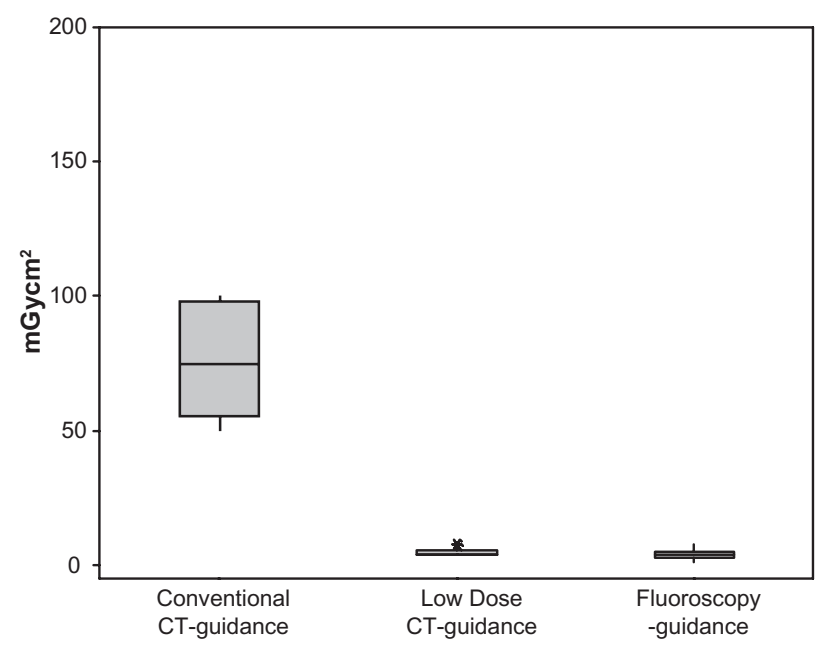

Figure 4A Comparison of radiation doses of conventional CT-guidance, low-dose protocol, and fluoroscopy in $\mathrm{Sl}$ joint injections.

Abbreviation: $\mathrm{CT}$, computer tomography.

pulsed-fluoroscopy group and the low-dose CT group with the Mann-Whitney $U$-test, no significant difference between the groups was found $(P=0.1055$, alpha level $=0.05)$. The technique was simple to perform, fast, and reproducible. After a short learning curve, intervention times (first scan to injection of medication) ranging 70-150 seconds were reached. Intra-articular needle positioning was achieved in all patients. Despite the decreased image quality, the injections could be performed even in obese patients and in patients with severe osteoporosis. These factors are usually limiting the radiation dose reduction in other spinal injections. There were no intra- or postprocedural complications.

\section{Discussion}

CT-guided SI joint injection radiation doses can be approximated to pulsed fluoroscopy levels using the

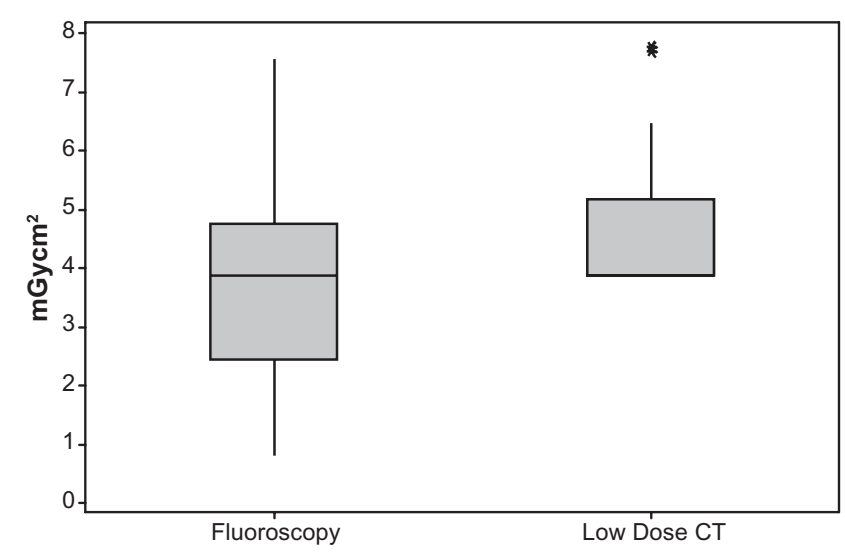

Figure 4B Comparison of radiation doses of fluoroscopic- and low-dose CT-guided SI-joint injections.

Abbreviation: CT, computer tomography. technique and protocol described above. The learning curve for this technique is fast, the technique is easy to perform, and reproducible. Mean radiation doses of $4.57 \mathrm{mGycm}^{2}$ (95\% CI: 4.13-8.24) were achieved. The available data from literature suggest different radiation dose exposures in spinal interventions. While Shepherd et al describe general radiation doses of $199 \mathrm{mGycm}^{2}$, Schmid et al calculated average radiation doses of 1.51-3.53 $\mathrm{mSv}$ (corresponding to 100.67$235.33 \mathrm{mGycm}^{2}$ ) for conventional CT-guided injections and $0.22-0.43 \mathrm{mSv}$ (corresponding to $14.67-28.67 \mathrm{mGycm}^{2}$ ) for low-dose CT-guided injections and $0.1 \mathrm{mSv}$ (corresponding to $6.67 \mathrm{mGycm}^{2}$ ) for pulsed-fluoroscopy-guided injections. ${ }^{14,15}$ Leng et al calculated average skin radiation doses of $195 \mathrm{mGy}$ for CT-guided injection procedures. ${ }^{16}$ Hendrix et al calculated radiation doses ranging between 12-30 mGy in fluoroscopyguided SI joint injections, while Vassiliev et al and Acho et al calculated mean skin radiation doses of $108 \mathrm{mGy}$ and $37.4 \mathrm{mGy}$, respectively, based on phantom studies in fluoroscopically guided SI joint injections. ${ }^{13,17,18}$ Compared to the protocol described in this study, a large reduction of the radiation dose could be achieved.

Despite the fact that most studies suggest that the level of fluoroscopy utilization is below a level of elevated concern, long-lasting effects of such radiation exposure are uncertain. ${ }^{19,20}$ Using fluoroscopy-guidance in spinal injections leads to radiation exposure of larger areas of the body in patients (back and pelvis) and the interventionalist (eg, hands, eyes, lower extremity). ${ }^{19-22}$ Radiation doses and exposure in CT-guided interventions are usually higher than in fluoroscopy-guided interventions. Due to the standardized use of fluoroscopy-guidance in SI joint injections in our department, the conventional CT-guidance was performed only in exceptional cases (eg, sacroiliitis, SI joint arthritis [five cases]), when strictly intra-articular application of medications was needed - therefore, the small number of five conventional CT-guided injections may be a possible limitation of this study. CT-guidance is, despite the higher exposure to radiation, still the most precise technique for intra-articular injections. Using the low-dose technique and protocol, significant reduction of radiation could be achieved, along with the benefit of precise needle positioning.

\section{Disclosure}

The authors report no conflicts of interest in this work.

\section{References}

1. Hansen HC, McKenzie-Brown AM, Cohen SP, Swicegood JR, Colson JD, Manchikanti L. Sacroiliac joint interventions: a systematic review. Pain Physician. 2007;10(1):165-184. 
2. Schwarzer AC, Aprill CN, Derby R, et al. The false-positive rate of uncontrolled diagnostic blocks of the lumbar zygapophysial joints. Pain. 1994;58:195-200.

3. van der Wurff P, Meyne W, Hagmeijer RH. Clinical tests of the sacroiliac joint. Manual Ther. 2000;5(2):89-96.

4. Riddle DL, Freburger JK. Evaluation of the presence of sacroiliac joint dysfunction using a combination of tests: a multicenter intertester reliability study. Phys Ther. 2002;82:772-781.

5. Dussault RG, Kaplan PA, Anderson MW. Fluoroscopy-guided sacroiliac joint injections. Radiology. 2000;214(1):273-277.

6. Hodge JC. Miscellaneous procedures: sacroiliac joint arthrography. In: Hodge JC, editor. Musculoskeletal imaging: diagnostic and therapeutic procedures. Basel, Switzerland: Karger Landes Systems, 1997:226-227.

7. Theodoridis T. Injection therapy of the spine without imaging. Der Orthopäde. 2007;36:73-86.

8. Braun J, Bollow M, Seyrekbasan F, et al. Computed tomography guided corticosteroid injection of the sacroiliac joint in patients with spondyloarthropathy with sacroiliitis: clinical outcome and follow-up by dynamic magnetic resonance imaging. J Rheumatol. 1996;23:659-664.

9. Cui Y, Xiao Z, Shuxia W, et al. Computed tomography guided intraarticular injection of etanercept in the sacroiliac joint is an effective mode of treatment of ankylosing spondylitis. Scand J Rheumatol. 2010 39(3):229-232.

10. Gupta S. Double needle technique: An alternative method for performing difficult sacroiliac joint injections. Pain Physician. 2011;14: 281-284.

11. Forst SL, Wheeler MT, Fortin JD, Vilenski JA. The sacroiliac joint Anatomy, physiology and clinical significance. Pain Physician. 2006;9: 61-68.

12. Walker JM. The sacroiliac joint: A critical review. Phys Ther. 1992;72: 903-916.
13. Hendrix RW, Lin PJ, Kane WJ. Simplified aspiration or injection technique for the sacro-iliac joint. Brief Note. J Bone Joint Surg. 1982; 64A(8):1249-1252.

14. Shepherd TM, Hess CP, Chin CT, Gould R, Dillon WP. Reducing Patient Radiation Dose during CT-Guided Procedures: Demonstration in Spinal Injections for Pain. Am J Neuroradiol. 2011;32(10):1776-1782.

15. Schmid G, Shmitz A, Borchardt D, et al. Effective Dose of CT- and Fluoroscopy-Guided Perineural/Epidural Injections of the Lumbar Spine: A Comparative Study. Cardiovasc Intervent Radiol. 2006;29:84-91.

16. Leng S, Christner JA, Carlson SK, et al. Radiation Dose Levels for Interventional CT Procedures. Am J Radiol. 2011;197(1):w97-w103.

17. Vassiliev D, Blackwood M, Kincel D. Patient entrance skin dose during three most common fluoroscopy-guided pain procedures. Reg Anesth Pain Med. 2003;28.

18. Acho S, van der Merwe B, van der Merwe D. Skin doses in fluoroscopically guided interventional procedures in back pain management. S Afr Radiogr. 2009;47(1):7-9.

19. Botwin KP, Thomas S, Gruber RD, et al. Radiation exposure of the spinal interventionalist performing fluoroscopically guided lumbar transforaminal epidural steroid injections. Arch Phys Med Rehab. 2002;83:697-701

20. Manchikanti L, Cash KA, Moss TL, Rivera J, Pampati VS. Risk of whole body radiation exposure and protective measures in fluoroscopically guided interventional techniques: A prospective evaluation. $B M C$ Anesthesiol. 2003;3(1):2.

21. Botwin KP, Freeman ED, Gruber RD, et al. Radiation exposure to the physician performing fluoroscopically guided caudal epidural steroid injections. Pain Physician. 2001;4:343-348.

22. Mahesh M. Fluoroscopy: patient radiation exposure issues. Radiographics. 2001;21(4):1033-1045.
Journal of Pain Research

\section{Publish your work in this journal}

The Journal of Pain Research is an international, peer-reviewed, open access, online journal that welcomes laboratory and clinical findings in the fields of pain research and the prevention and management of pain. Original research, reviews, symposium reports, hypothesis formation and commentaries are all considered for publication.

\section{Dovepress}

The manuscript management system is completely online and includes a very quick and fair peer-review system, which is all easy to use. Visit http://www.dovepress.com/testimonials.php to read real quotes from published authors. 